\title{
Tell me who you hang out with and I will tell you who you are
}

\author{
Valerio Rubino
}

September, 2019

\section{Introduction}

This report will revolve around a paper published in 2012 by Saygin [1], which combines diffusion and functional imaging to prove that the functionally-defined fusiform face area can be identified by a characteristic pattern of extrinsic connectivity. As the saying goes: "tell me who you hang out with and I will tell who you are". First, I will briefly review some proposals [2-4] about the relationship between connectivity and function to unravel the relevance of the study [1] at issue. Then, I will touch some issues related to structural connectivity data analysis [5] and how they have been overcome within a probabilistic framework [6]. Next, I will survey experiments [7-10] which built on the latter and to which Saygin's [1] added. In the following section, I will describe the methods and results of Saygin's [1] article, whose limitations and implications will be then explored in the discussion.

1.1 Connectivity and function A 1990 paper, published by Sur [2] opened with the question: "what is intrinsically visual about visual thalamus and cortex?". Such doubts were raised by discoveries from rewiring experiments on ferrets (that are equipped with remarkably immature brains at birth): researchers induced retinal ganglions to grow into the auditory thalamus (MGN), which relays signals to auditory cortex. In operated ferrets, rerouted visual afferent processes imparted visual function (i.e. visual receptive field properties)[2, 11]; then, what defines such auditory or visual cortices, if altering the afferentation of auditory cortex radically changes its development, to the point it exhibits strikingly functional resemblance with visual one? A guy with a clear-cut answer to these questions was Vernon Mountcastle; as for him, what differentiate entities in the brain (i.e. areas) is not the set of computations they carry out (which are instead repetitive and stereotyped functional units the columns, which map inputs to outputs through qualitatively similar computations and pervasively span the neocortex; these columns are linked together by their patterns of connections, constituting larger entities (i.e. brain areas), each with, due to their distinctive extrinsic connections, specific cytoarchitectonic and functional properties[3]. A similar but less radical proposal has been championed by Passingham [4], which formalized the "connectivity fingerprint" hypothesis, according to which each brain area has a unique set of extrinsic inputs and outputs which identify it, much like a fingerprint. As Passingham himself acknowledged [4], this hypothesis sounded anything but astonishing. Indeed, ten years before, Young [12, 13] laid the foundations of a research program which consists of analytical traction of large databases of tracer studies collated into structural connectivity matrices. Following his lead, this and similar approaches have been repeatedly reported to extract connectional structures which reflect the ones delineated by function [14-18]. Yet, Passingham's proposal was much bolder than that. He suggested that the alignment of connectional and functional architecture owes to the former determining it altogether with local processing features [4]; indeed, anatomical connections constraint the information available to an area and the influence it can exert area which are thought to correspond to the transformation it carries out $[4,9,19]$. Hitherto, it was not possible to prove this conjecture [14]: animals require extensive training and it is difficult to electrophysiologically record from many areas, precluding these theses to be tested whole-brain and on higher-level functions Nonetheless, Passingham envisioned the potential of combining diffusion and functional imaging to overcome this stalemate[4]. Yet, to use diffusion imaging to this end, there is a tough nut to crack.

1.2 Imaging aTo inform about connections, data so collected needs to go through two more steps: local modelling - which consists in mapping each voxel to its value within the chosen diffusion metrics, and fibre tracking (FT) algorithm - which, fed with the previously generated map, follow continuous paths between its point to reconstruct fibre tracts [20]. A common combination features Diffusion Tensor Imaging (DTI) and deterministic tractography. For each voxel, DTI estimates a tensor that models its degree of anisotropy and a single preferred direction of diffusion which, allegedly, is parallel to the bundle of axons it contains ([21, 22]). A deterministic FT algorithm (DT-FT), once fed with the so generated tensor map, iteratively compares the principal directions of a 
pair of neighbouring voxels and decide whether their fibres are likely to form a continuous bundle (i.e. they do not exceed a user-defined level of divergence); after initiating from a seed and running along so defined trajectories, it establishes the targets the former interconnects with [22]. Although popular, this approach has some flaws: as DWI is very susceptible to artefacts (e.g. [23]), conservative thresholding is required to avoid false-positives and unexpected behaviours; GM voxels contain intricate fibres and artifactually approach isotropy due to the averaging of superimposed multiple directions of diffusion. Due to thresholding, then, the baby (i.e. information residual to the simple tensor model) is thrown out with the baby water (i.e. noise); therefore, in the attempt to dig a streamline up to GM voxels, deterministic algorithms are brought to a halt. Indeed, these limitations plague, for example, a study by Vassal [24]; due to the low anisotropy of the grey-white matter interface, their deterministic FT algorithm could not achieve a finer-grained description of fibres terminations, limiting the scope of the analysis to major fascicles.

The probabilistic framework introduced by Behrens [6, 7] was then, truly, a game-changer. In his proposal, both local modelling and fibre tracing take care explicitly of the uncertainty of estimations (due to artifacts and inadequacy of the local model), associating probability distributions to free parameters of the models. The algorithm makes use of heuristics to draw samples from the probability distributions, effectively computing the probability each voxels are connected given the observed data. Therefore, algorithms of this kind can run even in the presence of low SNR and overcome shortcomings of deterministic tractography; in principle, this framework is compatible with any local modelling choice - both the aforementioned tensor and a simple partial volume model (which assumes the signal from each voxel is the sum of an isotropic and an anisotropic compartment); simple partial volume is conceptually more proper, because aims to more directly to characterize tissue microstructure rather than diffusivity profile. Nonetheless, they obtain similar results [6]. The local model can even be more complex, allowing each voxel to be composed by subvoxels of arbitrarily small size, each containing an axonal tract of idiosyncratic directions. It is important to stress that probabilistic and deterministic approaches are more of complementary sources of information than competitors: the former calculates our confidence about whether two voxels are connected, and output more numerous tracts, while the latter, looking only at the parameters which best fit the data, characterize more consistently the major WM tracts [20].

1.3 Background and motivation In the first reported display [7], probabilistic tractography was seeded from voxels of the thalamus to manually-segmented targets of interest; thalamic voxels were then classified according to the region they were more likely to connect with. The resulting mapping was consistent with known locations of thalamic nuclei; the approach was further validated by showing those nuclei overlap with functionally-defined regions in standard stereotactic space [9]. This hard segmentation approach was further refined in 2011 by Saygin and colleagues [10]: distinctive connectivity profiles, inferred from tracer studies on non-human models, were expressed through Boolean logic (i.e. negations, conjunctions and disjunctions) and then applied to connectivity distributions to differentiate amygdala in four nuclei. Yet, these attempts owe to previous knowledge about meaningful and diagnostic targets and thus have a rather limited scope [19]. Johansen-Berg and Behrens themselves published, in 2004, a study [8] introducing a little twist; connectivity profiles were correlated with each other to obtain a cross-correlation matrix, which was then algorithmically reordered and visually inspected for clusters - group of seed voxels with similar patterns of connections. The cortical focus of the study was medial frontal cortex (MFC) - BA 6, which appeared to be connectionally dissociated in two discrete and spatially contiguous regions along the posterior-anterior axis, which bear close resemblance with the centres of mass of pre-SMA and SMA proper revealed by functional contrasts (finger tapping vs. serial subtraction) collected within the same experiment; these results allow to draw two important inferences: first, it is possible to overcome the need to prespecify distinguishing targets to parcel brain regions; second, although blind to functional contrast, connectivity-defined boundaries align with functional selectivity in MFC. Again, it would not be feasible to generalize this approach to test connectivityfunction claim on the whole brain, as it relies on visual inspection of cross-correlation matrice and not on an automated algorithmic decision [19]. Another way to tackle the issue has been explored by Saygin [1] and will be discussed in details in the present report; it builds on the realization that if functionally-defined areas are associated with a distinctive pattern of connection, then there should be a mapping from connectivity distributions for a voxel to its functional activity. The authors specified this mapping as a linear combination, whose weights were estimated through least-square error regression. Although this is a rather simple model and thus a conservative stance, this proof of concept study succeeded. Jbabdi and Behrens [25] described this approach through an enlightening analogy: it is like inferring whether Tube station passengers are workers or tourists from their destinations or provenances respectively, business districts or touristic meccas. Saygin and colleagues [1] decided to focus on the fusiform face area (FFA), which is a region of the fusiform gyrus, in the ventral temporal cortex, which is activated by faces and thus regarded as domain-specific [26, e.g.]. 


\section{Methods and results}

2.1 Data acquisition and processing The sample was partitioned in two cohorts (of 23 and 21 participants each), which underwent DWI and fMRI in the same scanner but under two slightly different parameters instantiations. While DWI parameters for both groups featured isotropic $8 \mathrm{~mm}^{3}$ voxels and $b$-value of $700 \mathrm{~s} \mathrm{~mm}^{-2}$, and differed only in the numbers of sampled directions, the main difference between the two fMRI protocols laid in the choice of, respectively, event-related and block designs, and either pictures of faces and scenes or movie clips of faces, scenes, objects and bodies as stimuli.

The FT algorithm used can be contrasted with the one applied in an aforementioned study [24]. While Saygin's [1] local model allowed each voxel for multiple principal directions, Vassal's [24], being a tensor model, assumed a single principal direction. DT-FT requires the specification of an anisotropy threshold which was set, by Vassal and colleagues [24], at 0.20, while no such constraint is needed in the probabilistic framework. As a consequence, DT-FT must be seeded and terminated within WM and, therefore, two expedients are made necessary: i) establish cortical terminations in areas and not voxels and ii) spatial resolution must be scaled down to connect BOLD clusters and fascicles. While, in principle, probabilistic tractography could compute connection probabilities voxel-wise, such high-dimensional spaces would be computationally expensive to tractate. Moreover, it would be non-trivial to bring voxel-wise connection probabilities in a common currency across subject and still retain individual anatomical variations [27]. Therefore, Saygin [1] settled for determining the connection probabilities between each fusiform gyrus voxel and each of 85 automatically segmented brain areas. Still, the handle of uncertainty supplied by the probabilistic tractography framework allowed the authors to align functional and tractography data without any blurs.

\subsection{Linear regression}

2.2.1 Training and testing For each participant of the Group 1, every voxel belonging to the fusiform gyrus was associated with an 85-dimensional vector (whose elements are the connection probabilities between the voxel and each of the 85 regions) and a t-value emerged from the contrast faces $>$ scenes; these data were concatenated across subjects, leaving the model blind to participants' identities. Then, a linear regression was trained to assign coefficients to each of the elements of the connectivity vectors (the features) centre to minimize the square difference between the t-values (the targets) and the results of their weighted sum. The metric chosen by the authors the evaluate the performance of the linear model was the mean absolute error (MAE): the average unsigned difference between observed and predicted t-values. The practice to train and evaluate a model with the same data is referred to as double-dipping; a model eventually captures noise of the training examples and evaluating it with the same dataset would optimistically bias the accuracy estimation [28]; to avoid circularity without giving up to examples during training authors resorted to leave-one-out-cross-validation (LOOCV): the hypothesis is tuned to fit the data from 22 out of 23 participants, and then tested on the held-out one; this procedure is repeated iteratively, rotating across all participants and the resulting performances are then averaged.

\subsubsection{Benchmarks The results were compared against three controls.}

The brain is recognized as a small-world network and, as such, features low path length and high level of clusterization [29]. Therefore, neighbouring areas are more likely to be connected and connection probability between two voxels downscale as their distance increases; this confound, together with the known anatomical bias along the medial to lateral axis for, respectively, scenes and faces [30], make the conclusions spurious as it could be the case that the model owes its success on physical distance rather than on connectional information itself. To exclude this possibility, linear models were trained following the same procedures, but feeding it with the Euclidean distance of the fusiform gyrus voxel and the centres of mass of the 85 target regions. The performances of the two models, measured through MAE, revealed connectivity-based model outperformed the distance one in 21 out of 23 subjects at a threshold of $\mathrm{P}<0.001$.

To check those results were statistically significant, the authors performed a permutation test. Under the null hypothesis, predictors and labels do not bear any linear relationship and, therefore, it makes no difference to shuffle them across examples. Indeed, this is how a permutation approach empirically estimates the null distribution and overcomes the presumption of a generative model for the data: sampling a permutation of the labels indexes, pairing them with features randomly [31]. Linear regressions were trained and tested on 5000 such generated datasets, achieving a null distribution of performances; for 22 out of 23 participants, the accuracy of the model trained on genuine data was in the top five per cent of performances, proving that it is significantly higher than chance. 
The third benchmark was a group-average model, built and evaluated through a similar LOOCV routine. fMRI data from all the participants but one were was normalized into MNI space and superimposed to generate a $t$-statistic image; by registering it to the remaining participant's native space, the authors obtained a group averagedriven prediction of activity in the fusiform gyrus, which was compared with the actual pattern of activation to compute the MAE. The connectivity-based model won against the group-average one in 17 out of 23 participants.

2.2.3 Other results The model was also tested with data from the Group 2, providing a measure of how well it generalizes across different scanning parameters and protocols, after being trained with the complete 23 participants datasets. As expected, this analysis confirmed the findings reported above.

Each of the features (i.e. probabilities of connections) contribute to determining the functional selectivity output to an extent quantified by its regression weight; therefore, weights were surveyed to identify the most reliable predictors (i.e. those whose coefficients significantly exceeded 0). This analysis selected positive (diagnostic of face selectively) and negative (conversely, diagnostic of scene selectivity) predictors which, when served alone as learning yielded to significantly better predictions. This subset of targets contained neighbouring and, to a higher degree, distant regions; a linear regression fed with an even more down-sampled pool of features - excluding connection probabilities with proximal targets, achieved better-than-chance predictions as well, demonstrating that predictions did not rely only on connections to close target regions.

Lastly, the authors report an analysis of the location of best positive (i.e. right inferotemporal cortex) and negative predictors (i.e. right lingual cortex) within individual subjects' native spaces. They showed centres of functional activation and connectivity to inferotemporal cortex variations along the medial-lateral axis correlate: connectivity model grasps spatial variability and thus is more reliable than group-average ones.

2.3 Summary Saygin and colleagues [1] proved that a linear readout of the face sensitivity of a fusiform gyrus voxel is possible from its connection probabilities to other areas. Moreover, they showed these results cannot be due to chance or to distance information embedded in connections probabilities. The accuracy of the connectivity-based model outperformed the group average model; indeed, locations of face selective voxels vary along the medial-lateral axis and colocalize with voxels which are more likely to connect with face predictors; therefore, the connectivity model grasps individual variation which is instead smoothed by averaging across participants.

\section{Discussion}

Saygin's [1] results prove connectivity fingerprint hypothesis; importantly, it does not follow it holds at other levels of structural descriptions (i.e. meso- and microscale [32]) and finer-grained or higher functions. In the next three sections, I will discuss how technical advancements, as well as multi-methods and multi-tasks approaches, can allow to investigating the matter more in depth. In the last three, instead, I will discuss how the described findings relate to open questions - namely, the causative role of connectivity, modularity and cartography of the brain.

\subsection{Limitations}

3.1.1 Technical advancements The paradigm proposed by Saygin [1] could be advanced in three sensible ways. First, the authors call for the implementation of voxel-to-voxel tractography. Although it would dramatically increase the dimensionality of data (and, thereby, computational costs), it would characterize connectivity profiles at a finer grain. A challenge posed by this suggestion lies in inter-subject variability: individual voxels would need to undergo transformations to be brought in a common space [27].

Second, the authors stressed that the posited linear fit is a parsimonious and rather conservative choice: odds are that nonlinear hypotheses would be required to apply the approach to other areas or higher-level functions, because connectivity-function relationships could be way more complex.

Lastly, another technical improvement could regard local modelling - more complex diffusion-axons mappings would better describe tracts in cases of, for example, crossing fibres and increase the confidence in relating connection probabilities returned by tractography to probabilistic strengths which is controversial [33]. Yet, it is important to point out that local modelling choice is not simply a matter of accuracy versus cheapness trade-off. Rather, different levels of complexity supply different and complementary information [20]. 
3.1.2 Structural connectivity Probabilistic tractography, while rendering viable connections, does not resolve whether they are actually relevant in given functional contexts (i.e. face processing). This drawback, acknowledged by Saygin and colleagues [1], kept them from clarifying the reasons why connections with target regions predict sensitivity to face. For instance, the best predictors feature cerebellum cortices, which does not belong to the face-processing network. In this respect, Fries [34], while maintaining that anatomical connectedness impose a communication structure, argued that on top of it sits a dynamic structure, which allows flexible routing of signals. In other words, although structural connectivity constrains neural communication, a certain function may just one single realization of a one-to-many structure-function mapping [35]. Indeed, synaptic connections exhibit context-, activity-, and time-dependent variability [36]; thus, functional roles of connected areas can only be elucidated by evidence sensitive to dynamical changes; such requirement is met by functional connectivity, which is the coupling of the activity of remote neurons, quantified by statistical dependencies, e.g. pairwise correlations between voxels BOLD time-courses $[36,37]$. Indeed, the correlational structure of BOLD signals changes in different tasks - likely due to superposition of spontaneous and task-evoked activity [38], and can be altered: for example, Tagliazucchi [39] administered LSD and placebo to participants, which then underwent rest-fMRI to characterize correlations between spontaneously fluctuating BOLD signal in 401 ROIs; these measures were then used to compute the functional connectivity density (FCD) as the average correlation of an ROI time series with the ones of all the remaining ones - a metric of interconnectedness of a single ROIs with all the other ones; LSD administration changes FCD values in frontal, parietal and inferior temporal cortices, and bilateral thalamus, making them more tightly pair with sensory cortices. Furthermore, by using functional connectivity to label edges of a graph, the authors computed modularity (the extent modules of the systems have dense within- and sparse-between module connections) to show LSD assumption reduces intrinsic brain networks separation. While Tagliazucchi [39] performed analysis on 401 ROIs, tractation of the full voxelwise correlation matrix is, although computationally expensive, possible and allows to achieve finer-grained structures [40]. Apart from enabling the investigation of context-specific communication [35], functional connectivity supplies a graded measure of connectivity (e.g., correlation), while the mapping connection probability to strength is controversial [33]).

Furthermore, DWI-based structural imaging does not provide information to discriminate the polarity of fibre tracts and functional connectivity, as well, cannot elucidate the direction of information flow [36]. This is were effective connectivity kicks in: effective connectivity is the influence one system exerts over another and is measured by techniques such as Granger causality (GC) [36]. A promising application has been reported by Bastos and colleagues [41], who used directionality to distinguish between feedforward and feedback interactions in visual areas in monkeys; GC influences of a pair of areas determined the directed influence asymmetry index (DAI), which quantified how much a projection from a source area to a target was effectively feedforward, in a certain frequencies range. After collapsing across frequency bands, DAI of each region to every other region were used to establish a functional hierarchy, characterizing them with a feedforward/feedback score. This functional hierarchy correlated with a tracer study-derived anatomical one; furthermore, the authors reported that the functional hierarchy changes dynamically along epochs of the paradigms (i.e. changes feedforward/feedback balance of areas) and showed that effective connectivity can add task-related information to fixed structural descriptions of circuits. Indeed, in the context of face selectivity, a single voxel could be associated with a vector whose elements are the influences exerted by other areas on its time series during face processing; then, pattern classification methods could establish whether this effective connectivity fingerprint can predict voxels' selectivity. Yet, there are troubles in paradise. For instance, effective connectivity is subject to pitfalls, which can be alleviated by structural connectivity imposing biologically-plausible constraints to models [36].

The take-home message is that actual inflow and output is constrained but not entirely determined by structural connectivity; odds are that to resolve finer-grained functional descriptions anatomical, functional and effective connectivity complement each other and should be jointly integrated. For instance, cluster analysis like the one deployed by Johansen-Berg [9] can be used to establish correspondences across modalities [32].

3.1.3 Functional contrasts Above, I argued that tractography results are not influenced by tasks. Yet, this pertains connectivity data per se: in fact, Saygin's [1] tractation of them through linear regression is biased by the label it learned to predict (i.e. functional contrast). In a face $>$ scene contrast, faces and scenes regressors are pitted against each other, cancelling out shared steps of information processing. Whereas these steps are of no use in discrimination of faces and scenes, they may be necessary for both to be processed. This issue concealed important areas to neuroimaging investigation and calls for careful task analyses [42]; although it does not challenge connectivity fingerprint hypothesis, it poses serious obstacles to the project of explaining functions of an areas by their extrinsic connections: in the context of connectivity profiles classification, it may have obliterated regions which are important partners of FFA although they promiscuously interconnect with scene-selective voxels 
also. Indeed, the inferior frontal junction (IFJ), although absent from significant predictors, has been shown to communicate with FFA during face processing [43]. Baldauf and Desimone [43] presented participants with a stream of overlapping faces and scenes, flashed at two distinctive tag frequencies. Occasionally, participants were cued to attend one of the two stimuli. Beyond the unsurprising FFA engagement, IFJ exhibited a face tagging response as well, but only when faces were attended. Further analysis revealed that IFJ response is phase-shifted - reflecting the time for the signal to be relayed to frontal areas. More importantly, the authors reported a gamma-band coherence between IFJ and FFA, with the former driving the oscillation and the latter entraining it with a phase lag of $20 \mathrm{~ms}$. These results dovetail with the Communication Through Coherence hypothesis, which states that, to maximize their effectiveness, sending neurons bundle their spike into synchronized bursts which should be timed to arrive when the excitable window of receiver neurons is open; to do so, the two groups of neurons synchronize, with a phase lag which reflects conduction delays [34, 44]. Concerning Baldauf and Desimone's study, IFJ and FFA synchronize at gamma frequency to maximize the gain from signals fed back to FFA by IFJ, which the authors suggest to bias visual stream processing towards the attended stimulus. Although compelling, these findings do not entail IFJ-FFA interactions is necessary to face processing, because neuroimaging and correlational techniques and thus cannot rule out it is an epiphenomenal by-product. Such a service could only be accomplished by experimental manipulations of coherency (like for example, the approach illustrated by Polanía [45], who successfully showed that exogenous desynchronization of fronto-parietal circuits induced by tACS disrupts value-to-action transformation). Nonetheless, these results suggest it works in tandem with FFA and, although, IFJ is absent from predictors of face sensitivity. This incongruity owes to the kind of functional information the model is trained to predict: IFJ is similarly engaged by scenes [43] and, thus, connections with it fail to distinguish between face and scene selective voxels.

To characterize a more exhaustive list of partners for an area, three ways can be paved. A first alternative could be a data-driven approach, like the one in Johansen-Berg's [9] study. Another possibility is to identify predictors of selectivity in different functional contexts; indeed, this what Saygin and Osher themselves did in a subsequent experiment [27] which extended the one at issue in two important ways: first, they ran four functional localizers (i.e. faces, bodies, scenes, objects); second, linear regressions were trained to predict functional response in each of 148 parcels rather than only in the fusiform gyrus and then used the 148 148-dimensional vectors of weights to prove correlations between functional selectivity of an area and different flavours of its predictiveness. Pushing this logic even further, multiple functional localizers results could be handled by Boolean logic (similarity to the way Saygin [10] took care of amygdala connections), whose conjunctions, negations and disjunctions can support the expression of complex functional patterns. Lastly, representational similarity analysis [46, 47], a more direct marker of representational content, could enter the model as targets instead of t-values; indeed, a model could be trained to weight connectivity profiles of a voxel to predict the value correlation between its neural similarity structure (which can also be obtained voxel-wise, through adaptation - e.g. [48]) and a representational geometry of interest. To sum up, the findings should be interpreted with caution, because best predictors do not exhaust the list of partners FFA jointly works with IFJ during face processing; stronger inferences can be drawn from paradigms featuring multi-faceted contrasts, description of more complex functional patterns and more direct markers of representation content.

\subsection{Implications}

\subsubsection{Localizers}

3.2.2 Connectivity and function Although Saygin [1] showed that a linear regression can compute face sensitivity of voxels from their connectivity profiles is possible, it does not follow connectivity fingerprints determine functional properties. Although pattern classification methods cannot disambiguate between genuine, inverse and spurious causation, they may be of use to assess whether connectional structure precedes functional organization which is required by connectivity determining function. Saygin [49] ran a longitudinal study in this vein, focusing on VWFA (an area of fusiform gyrus sensitive to words). Children underwent diffusion-weighted and functional scanning at 5 and 8 years. By analyzing the resulting data in the previously described fashion, authors proved that properly weighted patterns of connectivity earmark fusiform voxels in non-reader brains which will acquire functional selectivity to words only after acquiring reading skills. These findings are consistent with connectivity profiles being precursors to reading acquisition and not vice versa[50]. Yet, while ruling out inverse causation, longitudinal studies do not exclude spurious correlation; indeed, microstructural features, which translate into local processing properties, delineate brain areas as well [19] and extrinsic connectivity could predict functional selectivity because they are proxies for other properties (e.g. biophysics, intrinsic connectivity). Comparisons of transcriptomic 
and connectional models could disentangle these possibilities. Transcriptomic allows describing the gene usage in anatomical structures [51], which determine both local processing properties and extrinsic connectivity. Linear regression methods, like the one applied by Saygin [1], can be used to study how much of function variability is explained by connectivity: if other structural features contribute little to determine activity, then they will be seen as noise by the model and get assigned low weights. Of course, this prediction is rather far-fetched and unlikely; yet, this approach could help elucidate the extent different end-points of genes expressions (e.g. extrinsic and intrinsic connectivity) relate to function.

3.2.3 Modularity Functional segregation (or specialization) and functional integration are two frameworks which have separately investigated the brain under distinct assumptions: respectively, that functions can be mapped to distinct areas and that, instead, functions arise from interactions [25, 32, 36]. Whereas this dichotomy dates back to the XIX century quarrel between localizationism and connectionism [36], the discussed paper calls for a bridge between the two positions [25]: brain areas are specialized by virtue of the other segregated areas they contact and thereby the information they integrate. It is perhaps worth to point out the two positions are not contradictory. Indeed, for an area to be domain-specific it is demanded to be optimized to perform a certain task [26]; for example, diverse computations are implemented through specific shapes of connections (i.e. convergent and divergent) [30]. Another proposal which understands functional segregation in light of functional integration (and vice versa) is the Distributed Domain-Specific Hypothesis [52], which posits that specialized networks to carry out specific ("eccentric") functions by contacting other segregated areas, according to an innate profile of extrinsic connectivity.

Segregation and integration can jointly be investigated employing of, for example, graph theoretical analysis, which has been briefly touched above in the context of Tagliazucchi's [27] study and was also applied by aforementioned Osher and Saygin's [27].

3.2.4 Patches and natural kinds "[T]o understand how a car works, one might first dissect the system into separate functional components such as pistons, wheels, or the carburetor; importantly, one next follows the cables and hoses to see what these components are connected with and how they come together to work in tandem" [53, p. 9]. It is self-evident that connectivity subserves the latter subtask - namely, characterizing interactions. Surprisingly, it may aid the former as well - again, defining and finding the elements of the network [32]. The quick survey of literature delineated in the introduction, along with Saygin's $[1,27]$ results demonstrate that connectivity parses the brain in regions exhibiting distinctive functional fingerprints. Other studies, as well, showed that connectivity space restructures functional properties meaningfully, ordering otherwise spatially scattered functional peaks (for a review, see [14]). It is not necessary to submit to a strong connectivity hypothesis like Passingham's [4] to see the exciting potential of these lines of research to make sense of cortex much like cartography [37]. This challenge resembles search for natural kinds - which are the entities in which nature should be carved to enable generalization [54]. The coincidence of functional, connectional and local processing profiles fits within the Homeostatic Properties Clusters (HPC) theory, which suggests natural kinds are defined by bundles of features, retained together by causal mechanisms (either intrinsic or extrinsic to them) [55]; in light of this perspective, best candidates for robust generalizations are those brain regions defined basing on multiple properties - in this context, functional activity, structural, functional and effective connectivity.

\section{Conclusions}

The study [1] which the present report revolved around is a proof of principle; the principle is that connectivity fingerprints earmark functional areas [4]; the method to demonstrate it is the most straightforward: to check whether a properly weighted sum of connection probabilities from a voxel to other parcels of the brain results can predict its functional activity. The chosen testbed is FFA, a famous area with well-known specific and non low-level functional specificity. Nonetheless, not only the results confirmed connectivity fingerprint hypothesis, but offer numerous novel insights: for instance, they call for a bridge between functional segregation and integration [25] and show the potential of developing fast connectivity-based localizers of regions of interest, which in turn would increase generalizability and statistical soundness of neuroimaging results through the definition $a$ priori of regions of interest $[28,56]$.

The bad news is that the study cannot test Passingham's [4] original claim, according to which connectivity profiles reflect functional properties because the former exerts a causal influence on the latter. Nonetheless, the approach introduced by Saygin [1] is fruitful and affords extensions along several directions. I described some which 
have been already explored (i.e. prediction of other categories sensitivity [27] and longitudinal study of connectional patterns and functional specialization of VWFA [49]) and others which are yet to come.

The most exciting of these future directions are, perhaps, multi-tasks, multi-methods experiments, encompassing multiple forms of connectivity and other structural features. Across the whole report, I touched many proposals, whose common theme is that cognition arises from the integration of diverse and interconnected groups of neurons $[25,32,36,52]$; perhaps, the elective approach to understanding it cannot be anything but a collaborative effort orchestrating different techniques, each with the "eccentric" and specific information they provide.

\section{References}

1. Saygin, Z. et al. Anatomical connectivity patterns predict face selectivity in the fusiform gyrus. Nature neuroscience 15, 321-7 (2011).

2. Sur, M. et al. Experimentally induced visual projections into auditory thalamus and cortex. Science 242 4884, 1437-41 (1988).

3. Edelman, G. M. et al. The mindful brain : cortical organization and the group-selective theory of higher brain function / Gerald M. Edelman, Vernon B. Mountcastle; introd. by Francis O. Schmitt (MIT Press Cambridge, 1978).

4. Passingham, R. E. et al. The anatomical basis of functional localization in the cortex. Nature Reviews Neuroscience 3, 606-16 (2002).

5. Jones, D. K. et al. Non-invasive assessment of axonal fiber connectivity in the human brain via diffusion tensor MRI. Magnetic resonance in medicine 42 1, 37-41 (1999).

6. Behrens, T. E. J. et al. Characterization and propagation of uncertainty in diffusion-weighted MR imaging. Magnetic resonance in medicine $\mathbf{5 0}$ 5, 1077-88 (2003).

7. Behrens, T. et al. Non-invasive mapping of connections between human thalamus and cortex using diffusion imaging. Nature neuroscience 6, 750-7 (2003).

8. Johansen-Berg, H. et al. Changes in connectivity profiles define functionally distinct regions in human medial frontal cortex. Proceedings of the National Academy of Sciences of the United States of America 10136 , 13335-40 (2004).

9. Johansen-Berg, H. et al. Functional-Anatomical Validation and Individual Variation of Diffusion Tractographybased Segmentation of the Human Thalamus. Cerebral cortex (New York, N.Y. : 1991) 15, 31-9 (2005).

10. Saygin, Z. et al. Connectivity-based segmentation of human amygdala nuclei using probabilistic tractography. NeuroImage 56, 1353-61 (2011).

11. Sur, M. et al. Cross-modal plasticity in cortical development: differentiation and specification of sensory neocortex. Trends in Neurosciences 13, 227-33 (1990).

12. Young, M. Objective analysis of the topological organization of the primate cortical visual system. Nature 358, 152-5 (1992).

13. Young, M. et al. Brain structure-function relationships: Advances from neuroinformatics. Philosophical transactions of the Royal Society of London. Series B, Biological sciences 355, 3-6 (2000).

14. Mars, R. B. et al. Connectivity Fingerprints: From Areal Descriptions to Abstract Spaces. Trends in Cognitive Sciences 22, 1026-37 (2018).

15. Scannell, J. W. et al. The connectional organization of neural systems in the cat cerebral cortex. Current Biology 3, 191-200 (1993).

16. Burns, G. et al. Analysis of the connectional organization of neural systems associated with the hippocampus in rats. Philosophical transactions of the Royal Society of London. Series B, Biological sciences 355, 55-70 (2000).

17. Bozkurt, A. et al. Organization of primate amygdalo-prefrontal projections. Neurocomputing 38-40, 11351140 (2001).

18. Kötter, R. et al. Multimodal characterisation of cortical areas by multivariate analyses of receptor binding and connectivity data. Anatomy and embryology 204, 333-50 (2001).

19. Behrens, T. et al. Relating connectional architecture to grey matter function using diffusion imaging. Philosophical Transactions of the Royal Society B: Biological Sciences 360, 903-911 (2005).

20. Yo, T.-S. et al. Quantifying Brain Connectivity: A Comparative Tractography Study. Medical image computing and computer-assisted intervention : MICCAI ... International Conference on Medical Image Computing and Computer-Assisted Intervention 12 Pt 1, 886-93 (2009). 
21. Mori, S. et al. Principles of Diffusion Tensor Imaging and Its Applications to Basic Neuroscience Research. Neuron 51, 527-539 (2006).

22. Mukherjee, P. et al. Diffusion tensor MR imaging and fiber tractography: theoretic underpinnings. AJNR. American journal of neuroradiology 29 4, 632-41 (2008).

23. Soares, J. et al. A hitchhiker's guide to Diffusion Tensor Imaging. Frontiers in neuroscience 7, 31 (2013).

24. Vassal, F. et al. Combined DTI Tractography and Functional MRI Study of the Language Connectome in Healthy Volunteers: Extensive Mapping of White Matter Fascicles and Cortical Activations. PloS one 11, e0152614 (2016).

25. Jbabdi, S. et al. Specialization: The connections have it. Nature neuroscience 15, 171-2 (2012).

26. Kanwisher, N. Domain Specificity in Face Perception. Nature neuroscience 3, 759-63 (2000).

27. Osher, D. E. et al. Structural Connectivity Fingerprints Predict Cortical Selectivity for Multiple Visual Categories across Cortex. Cerebral cortex 26 4, 1668-83 (2016).

28. Kriegeskorte, N. et al. Circular analysis in systems neuroscience: The dangers of double dipping. Nature neuroscience 12, 535-40 (2009).

29. Zamora-López, G. et al. Exploring Brain Function from Anatomical Connectivity. Frontiers in neuroscience 5 (2011).

30. Grill-Spector, K. et al. The functional architecture of the ventral temporal cortex and its role in categorization. Nature reviews. Neuroscience 15 (2014).

31. Golland, P. et al. Permutation Tests for Classification in Proceedings of the 18th Annual Conference on Learning Theory (Springer-Verlag, Bertinoro, Italy, 2005), 501-15.

32. Sporns, O. et al. The Human Connectome: A Structural Description of the Human Brain. PLoS Computational Biology 1, 1111-20 (2005).

33. Jbabdi, S. et al. Tractography: Where Do We Go from Here? Brain connectivity 1 3, 169-83 (2011).

34. Fries, P. A mechanism for cognitive dynamics: neuronal communication through neuronal coherence. Trends in Cognitive Sciences 9, 474-480 (2005).

35. Park, H.-J. et al. Structural and functional brain networks: from connections to cognition. Science $\mathbf{3 4 2} \mathbf{6 1 5 8}$, 1238411 (2013).

36. Friston, K. Functional and Effective Connectivity: A Review. Brain connectivity 1, 13-36 (2011).

37. Sporns, O. Cerebral cartography and connectomics. Philosophical transactions of the Royal Society of London. Series B, Biological sciences $\mathbf{3 7 0}$ (2015).

38. Fox, M. et al. Spontaneous Fluctuations in Brain Activity Observed with Functional Magnetic Resonance Imaging. Nature reviews. Neuroscience 8, 700-11 (2007).

39. Tagliazucchi, E. et al. Increased Global Functional Connectivity Correlates with LSD-Induced Ego Dissolution. Current Biology 26, 1042-50 (2016).

40. Turk-Browne, N. Functional Interactions as Big Data in the Human Brain. Science (New York, N.Y.) 342, 580-4 (2013).

41. Bastos, A. M. et al. Visual Areas Exert Feedforward and Feedback Influences through Distinct Frequency Channels. Neuron 85, 390-401 (2015).

42. Bechtel, W. Aligning Multiple Research Techniques in Cognitive Neuroscience: Why Is It Important? Philosophy of Science 69 (2002).

43. Baldauf, D. et al. Neural Mechanisms of Object-Based Attention. Science (New York, N.Y.) 344, 424-7 (2014).

44. Bastos, A. M. et al. Communication through coherence with inter-areal delays. Current Opinion in Neurobiology 31. SI: Brain rhythms and dynamic coordination, 173-180 (2015).

45. Polanía, R. et al. The precision of value-based choices depends causally on fronto-parietal phase coupling. Nature communications 6, 8090 (2015).

46. Kriegeskorte, N. et al. Representational Similarity Analysis - Connecting the Branches of Systems Neuroscience. Frontiers in systems neuroscience 2, 4 (2008).

47. Kriegeskorte, N. et al. Representational geometry: Integrating cognition, computation, and the brain. Trends in cognitive sciences 17 (2013).

48. Garvert, M. et al. A map of abstract relational knowledge in the human hippocampal-entorhinal cortex. eLife 6 (2017).

49. Saygin, Z. et al. Connectivity precedes function in the development of the visual word form area. Nature Neuroscience 19 (2016). 
50. Dehaene, S. et al. Is the brain prewired for letters? Nature Neuroscience 19, 1192-1193 (2016).

51. Zeng, H. et al. Neuronal cell-type classification: Challenges, opportunities and the path forward. Nature Reviews Neuroscience 18 (2017).

52. Mahon, B. et al. What drives the organization of object knowledge in the brain? Trends in cognitive sciences 15, 97-103 (2011).

53. Saygin, Z. Structure-function relationships in human brain development PhD thesis (Massachusetts Institute of Technology, https://dspace.mit.edu/handle/1721.1/77843, 2012).

54. Slater, M. H. et al. in Carving Nature at its Joints: Natural Kinds in Metaphysics and Science (eds Campbell, J. K. et al.) (MIT Press, 2013).

55. Craver, C. F. Mechanisms and Natural Kinds. Philosophical Psychology 22, 575-94 (2009).

56. Saxe, R. et al. Divide and conquer: A defense of functional localizers. NeuroImage 30, $1088-96$ (2006). 